\title{
Aspectos cronobiológicos do ciclo vigília-sono e níveis de ansiedade dos enfermeiros nos diferentes turnos de trabalho*
}

\author{
CHRONOBIOLOGYC ASPECTS OF SLEEP-WAKE CYCLE AND ANXIETY LEVELS \\ OF NURSES WORKING ON DIFFERENT SHIFTS
}

LOS ASPECTOS DE CHRONOBIOLOGYC DE CICLO DEL DORMIR-ESTELA Y ANSIEDAD
NIVELAN DE ENFERMERAS QUE TRABAJAN EN LOS CAMBIOS DIFERENTES

Maria Luiza Pesse Campos', Milva Maria Figueiredo De Martino²

\section{RESUMO}

Esta pesquisa teve como objetivos: identificar os cronótipos dos enfermeiros dos diferentes turnos, estudar os padrões de sono e identificar os níveis de ansiedade estado-traço, correlacionando os cronótipos com as variáveis ansiedade-traço e ansiedade-estado. Foi realizado no Hospital de Clínicas da Universidade Estadual de Campinas (UNICAMP), com 40 sujeitos. O cronótipo preponderante dos enfermeiros do turno matutino foi o matutino moderado e os dos turnos vespertino e noturno do tipo indiferente. Em relação aos padrões de sono, os enfermeiros do turno matutino dormem menos, acordam mais cedo com ajuda do despertador em relação aos outros turnos. Quanto aos níveis de ansiedade traçoestado, situaram-se nos níveis I (baixo) e II (moderado) de ansiedade traço-estado. Este estudo permitiu que concluíssemos que os enfermeiros encontram-se satisfeitos e motivados com as condições de trabalho da instituição.

\section{DESCRITORES}

Ritmo cicardiano.

Trabalho em turnos.

Ansiedade.

Enfermagem

\begin{abstract}
This research had as objectives: to identify the chronotypes of nurses on different shifts, to study the sleep patterns and to identify the levels of anxiety trace-state, correlating the chronotypes with the variables trace-anxiety and state-anxiety. It was conducted at the Clinics Hospital of the State University of Campinas (UNICAMP), with 40 subjects. The prepon-derant chronotype of the morning shift nurses was the morning moderate and the ones from the afternoon and night shifts were the indifferent type. Regarding to the sleep patterns, the morning shift nurses sleep less, wake up earlier with help of the alarm clock in relation to the other shifts. As for the levels of trace-state anxiety, they were located in the levels I (low) and II (moderate) of trace-state anxiety. This study allowed us to conclude that the nurses are satisfied and motivated with the work conditions of the institution.
\end{abstract}

\section{DESCRIPTORS}

Cicardian rhythm.

Shift work.

Anxiety.

Nursing.

\section{RESUMEN}

Esta investigación tuvo como objetivos: identificar los cronotipos de los enfermeros de los diferentes turnos, estudiar los patrones de sueño e identificar los niveles de ansiedad estado trazo correlacionando los cronotipos con las variables trazo de ansiedad y ansiedad - estado. Fue realizado en el Hospital de Clínicas de la Universidad Estatal de Campinas(UNICAMP), con 40 sujetos. El cronotipo preponderante de los enfermeros del turno matutino fue el matutino moderado y los de los turnos vespertino y nocturno del tipo indiferente. En relación a los patrones de sueño, los enfermeros del turno matutino duermen menos, se despiertan más temprano con ayuda del despertador en relación a los otros turnos. En cuanto a los niveles de ansiedad de trazo estado, se situaron em los niveles I (bajo) y II (moderado) de ansiedad de trazo estado. Este estudio permitió que concluyamos que los enfermeros se encuentran satisfechos y motivados con las condiciones de trabajo de la institución.

\section{DESCRIPTORES}

Ritmo circadiano.

Trabajo en turnos.

Ansiedad.

Enfermería.

\footnotetext{
* Extraído da Dissertação "Aspectos cronobiológicos do ciclo vigíliasono e níveis de ansiedade dos enfermeiros nos diferentes turnos de trabalho", Faculdade de Ciências Médicas da Universidade de Campinas (FCM/ UNICAMP), 2002

1 Mestre em Enfermagem. Docente do Colégio Técnico da UNICAMP. malu@bol.com.br

2 Professora Doutora do Departamento de Enfermagem da FCM/ UNICAMP. Orientadora desta pesquisa.
} 


\section{INTRODUÇÃO}

O trabalho realizado em turnos, principalmente o trabalho noturno fixo ou alternante traz prejuízos à saúde do trabalhador e sob a óptica da cronobiologia estes prejuízos são decorrentes de uma desordem temporal do organismo. Após dois anos e sete meses de trabalho no turno noturno e tentando me adaptar a este horário, iniciei com episódios repetidos de faringite, infecções urinárias, distúrbios no padrão de sono, os quais creio, decorrentes de baixa resistência devido ao meu trabalho.

Solicitei a minha transferência para o turno vespertino e logo a seguir para o turno matutino. Acabaram as faringites, as infecções urinárias, os distúrbios no padrão de sono, porém surgiram novas dificuldades. Tinha uma escala a seguir e os plantões nos finaisde-semana não permitiam que eu compartilhasse da convivência familiar, o meu momento de lazer ficava prejudicado e isto era fonte de angústia, insatisfação e ansiedade.

Ao falar de minhas insatisfações com minhas colegas, estas também relatavam as suas, no entanto, percebia que algumas se mostravam mais adaptadas que as outras ao trabalho em turnos. Afinal, por que algumas pessoas se adaptam melhor que as outras ao trabalho em turnos?

Em busca destas respostas, interessei-me por assuntos relacionados à adaptação das pessoas aos turnos de trabalho, tais como estudos em cronobiologia e pesquisas sobre a presença de ansiedade nos enfermeiros, pois acreditava, por experiência profissional própria, estarem relacionadas ao trabalho em turnos.

Em levantamento bibliográfico realizado, "a cronobiologia pode ser entendida como o estudo sistemático da organização temporal da matéria viva" ${ }^{\prime \prime}$.

Segundo esses autores, detecta-se a organização temporal dos seres vivos, através de ciclos regulares na sua função, como exemplo o ciclo vigília-sono, que se repete aproximadamente a cada 24 horas.

O ciclo vigília-sono é um ritmo biológico que possui caráter endógeno, determinado geneticamente e sincronizado por pistas temporais. A natureza endógena dos ritmos biológicos aponta para a existência de estrutu- ras que são capazes de propiciar ao organismo esta ritmicidade, são os chamados relógios biológicos ou osciladores endógenos ${ }^{(2-3)}$.

Tais ciclos funcionam independente da exposição dos organismos aos ciclos ambientais, porém são influenciados por sincronizadores externos ou "Zeitgebers". O fenômeno claro-escuro é um dos sincronizadores mais potentes para os seres vivos. Para o homem, animal social, além deste, outro grande sincronizador é o de natureza social, formalizadores de hábitos sociais para a atividade diurna e repouso durante a noite ${ }^{(2,4)}$.

No trabalho em turnos, cuja maneira de se organizar a jornada seria em diferentes horários ou em horário constante, porém incomum, um exemplo é o trabalho noturno permanente, o indivíduo é obrigado a inverter o seu horário de dormir, causando uma desordem temporal do organismo e ao longo do tempo trazendo prejuízos à sua saúde ${ }^{(5-7)}$.

Os distúrbios do padrão de sono são sintomas preocupantes para os trabalhadores em turnos, o trabalhador terá um bom desempenho profissional se começar o seu trabalho descansado ${ }^{(6)}$. Isto para a enfermagem é fundamental, por tratar-se de uma profissão, cujo cuidado à pessoa não admite erros.

A desordem da estrutura dos ritmos circadianos causam mal-estar, fadiga, sonolência, insônia, irritabilidade, prejuízo da agilidade mental, desempenho e eficiência. O sono diurno é prejudicado pelas condições ambientais não favoráveis como iluminação, ruídos e acontecimentos domésticos, modificando a distribuição das fases do sono e interferindo em sua propriedade restauradora ${ }^{(4,7-8)}$.

A dessincronização dos ritmos circadianos a longo prazo pode levar a distúrbios severos e persistentes do sono como fadiga crônica e síndrome psiconeurótica (ansie-dade e depressão crônica), requerendo tratamento com drogas psicotrópicas ou hipnóticas ${ }^{(7)}$.

A ansiedade, que poderá estar presente nos enfermeiros por desordem dos ritmos circadianos, também foi identificada em estudo com enfermeiras inglesas que possuíam alto grau de ansiedade por lidarem com a dor, morte e o sofrimento ${ }^{(9)}$.

A ansiedade é diferenciada em dois conceitos, ansiedade-estado (A-estado) e ansiedade-traço (A-traço) $)^{(10)}$. 
A ansiedade-estado é uma condição/estado transitório do organismo humano. A ansiedade-traço refere-se a diferenças individuais, na tendência em reagir a situações percebidas como ameaçadoras, apresentando elevação no estado de ansiedade.

As pessoas vivem ansiosas porque não sabem o papel que deverão assumir e o enfermeiro é o profissional que vivencia estas situações em seu ambiente laboral ${ }^{(11)}$.

Tentando contribuir com o bem-estar dos indivíduos em seu ambiente laboral, alguns estudos foram realizados com o objetivo de buscar respostas a respeito da adaptação das pessoas aos turnos de trabalho ${ }^{(12-14)}$.

Estudos realizados mostraram que quando os enfermeiros escolhiam o esquema de trabalho que estava de acordo com sua programação de vida pessoal, o trabalho noturno tornava-se mais tolerável ${ }^{(15)}$. Tais resultados foram corroborados pela autora ${ }^{(16)}$.

O cronótipo ou tipo cronobiológico que diz respeito as diferenças individuais em relação à alocação dos períodos de sono e vigília nas 24 horas, compatível com o horário de sua atividade laboral parece ajudar os indivíduos na adaptação aos turnos de trabalho. A população humana pode ser dividida em três tipos básicos: matutinos; são pessoas que possuem preferência para dormir e acordar cedo e constituem 10 a $12 \%$ da população. Vespertinos: despertam tarde e dormem tarde e o seu melhor desempenho para o trabalho se dá no período da tarde ou à noite. Indivíduos deste tipo constituem oito a dez por cento da população e Indiferentes: não há preferência de horário para dormir e acordar e estes são mais tolerantes aos estados de privação de sono. Constituem a maior parte da população ${ }^{(17)}$.

Estudos cronobiológicos concluíram que indivíduos com hábitos vespertinos mais acentuados seriam os mais bem sucedidos para trabalhar à noite do que as pessoas com preferências matutinas ${ }^{(8,18)}$.

Diante destas considerações e na tentativa de buscarmos novos conhecimentos acerca do assunto, esta pesquisa teve como objetivos:

- Identificar os cronótipos dos enfermeiros que trabalham em turnos diurno e noturno;
- Estudar os padrões de sono através das variáveis: horários de dormir e acordar, latência do sono, qualidade e quantidade de sono diurno e noturno, maneiras de acordar e hábitos de cochilo dos enfermeiros;

- Identificar os níveis de ansiedade traçoestado (IDATE);

- Correlacionar o cronótipo com as variáveis ansiedade-traço e ansiedade-estado.

\section{MATERIAL E MÉTODO}

\section{Local e regime de trabalho}

Esta pesquisa foi realizada no Hospital de Clínicas da Universidade Estadual de Campinas (HC-UNICAMP), na cidade de Campinas, São Paulo.

O regime de trabalho dos enfermeiros é de jornadas de trabalho de seis horas diárias para os períodos matutino e vespertino, com dez folgas mensais. Para o turno noturno a jornada é de doze horas de trabalho por sessenta horas de folga.

\section{Sujeitos}

Participaram, voluntariamente, 40 enfermeiros sadios dos três turnos existentes, com idade entre 25 e 57 anos, de ambos os sexos.

\section{Critérios para seleção dos sujeitos}

Critérios de inclusão: Pertencerem ao quadro de pessoal do Hospital de Clínicas da UNICAMP e trabalharem em sistemas de turnos prestando assistência de enfermagem direta aos pacientes.

Critérios de exclusão: Possuírem mais de um vínculo empregatício, ocupando cargos administrativos e os inseridos em outras áreas, tais como: lavanderia, serviços gerais, áreas de apoio e comissão de controle de infecção hospitalar.

\section{Instrumentos}

- Ficha para caracterização dos sujeitos.

- Questionário de identificação de indivíduos matutinos e vespertinos ${ }^{17)}$, validado e adaptado às características e hábitos de nossa população ${ }^{(19)}$.

- Inventário de ansiedade traço-estado $(\text { IDATE })^{(10)}$, traduzido e adaptado à realidade brasileira $^{(20)}$.
Aspectos cronobiológicos

do ciclo vigília-sono

e níveis de ansiedade

dos enfermeiros

nos diferentes

turnos de trabalho 
Maria Luiza Pesse Campos Milva Maria F. De Martino
- Avaliação do ciclo vigília-sono elaborado e validado pelo Grupo Multidisciplinar de Desenvolvimento e Ritmos Biológicos do Instituto de Ciências Biomédicas da Universidade de São Paulo - ICB, USP(21).

\section{Aspectos éticos}

Antes de iniciar a coleta de dados, o projeto deste estudo foi encaminhado à Comissão de Ética em Pesquisa da Faculdade de Ciências Médicas da UNICAMP para avaliação, sendo aprovado.

Os sujeitos da pesquisa assinaram um termo de consentimento livre e esclarecido para participarem do estudo.

\section{Coleta de dados e tratamento estatístico}

A coleta de dados ocorreu durante o período de 08 de janeiro a 16 de fevereiro de 2001, até serem completadas as respostas dos 40 sujeitos constantes do estudo.

Para a análise da relação entre duas variáveis categóricas, foi utilizado o Teste Exato de Fisher e para variáveis em 3 ou mais categorias o Teste de Kruskal-Wallis. O nível de significância adotado foi de $5 \%$, ou seja, $\mathrm{p}<0,05$. Programa computacional utilizado: The SAS System for Windows (Statistical Analysis System), versão 6.12.SAS Institute Inc, 1989-1996, Cary, NC, USA.

\section{RESULTADOS E DISCUSSÃO}

Após estabelecermos os critérios de inclusão e exclusão, selecionamos 40 enfermeiros assistenciais que trabalham em sistemas de turnos fixos, matutino, vespertino e noturno, respectivamente: $n=14,11$ e 15 . A maioria dos sujeitos foi composta por mulheres (95\%). A faixa etária variou de 25 a 57 anos e a maioria (47,50\%) encontra-se na faixa etária de 31 a 40 anos, 60\% são casados, 65\% não tem filhos e $97,5 \%$ não utilizam medicamentos para dormir.

Observou-se que os turnos são homogêneos em relação à idade, não havendo diferença significativa para a idade, tempo de serviço e tempo de formado, embora exista uma tendência do turno matutino possuir profissionais com menos tempo de serviço. Não houve diferença significativa quanto ao número de plantões extras realizados entre os turnos de trabalho.
Em relação à análise dos parâmetros numéricos que caracterizam os episódios de sono, de acordo com os turnos de trabalho, houve diferença significativa na hora de acordar. Os enfermeiros do turno da manhã acordaram mais cedo do que os enfermeiros dos demais turnos (Teste de Kruskal-Wallis: $\mathrm{p}=0,0001$ ). Apesar de acordarem mais cedo, não anteciparam significativamente a hora de se deitarem. Achados semelhantes foram encontrados em estudo com enfermeiras que trabalhavam em turnos fixos ${ }^{(16)}$.

As médias ao turno matutino mostraram diferenças significativas para o duração do sono noturno entre os turnos (Teste de Kruskal-Wallis: $\mathrm{p}=0,0002$ ). Os enfermeiros do turno matutino tiveram duração de sono noturno menor que os dos demais turnos, talvez, por serem relativamente jovens, com idade média de 34,07 anos, que por método de vida social e familiar, acabam por dormir tarde, necessitando acordar cedo para o plantão.

Não houve diferença significativa na qualidade de sono, entre os turnos $(\mathrm{p}=0,8550)$. Observa-se que a qualidade do sono noturno não foi prejudicada pelo trabalho em turnos, talvez porque os enfermeiros apresentam idade média de 37,18 anos. As dificuldades para o trabalho em horários anormais aumentam à medida que os trabalhadores envelhecem ${ }^{(22)}$.

Verifica-se que os enfermeiros do turno da manhã são acordados em maioria por despertador, enquanto que os dos demais turnos acordam sozinhos $\mathrm{p}=0,010$. Dados que confirmam as características peculiares dos enfermeiros do turno matutino, que são obrigados a acordar em um horário diferenciado para o inicio de suas atividades.

Houve diferença significativa na percentagem de dias em que os enfermeiros cochilaram (Teste de Kruskal-Wallis: $\mathrm{p}=0,0005$ ). Para o grupo do turno matutino, os valores médios foram maiores.

Os resultados revelaram que para este grupo, a freqüência para o cochilo ou sesta à tarde foi maior. O cochilo é um tipo de sono que possui como característica ser um sono diurno mais curto que o do período noturno habitual $^{(23)}$. Os cochilos podem sugerir cansaço e fadiga devido à privação de sono ${ }^{(24)}$. Pode-se inferir que os cochilos sejam uma forma de minimizar um possível débito de sono. 
Na correlação dos grupos dos diferentes turnos com o cronótipo e níveis de ansiedade, os enfermeiros pertencentes ao turno matutino cujo cronótipo predominante foi o matutino moderado, obtiveram respostas de níveis de ansiedade traço-estado, nível II ou seja, nível moderado de ansiedade. Os enfermeiros pertencentes aos turnos vespertino e noturno foram classificados como do tipo Indiferentes. No entanto, quanto aos níveis de ansiedade estes foram diferentes, os enfermeiros do grupo vespertino demonstraram para o nível de ansiedade-traço e estado escores II (moderado) os do grupo noturno demonstraram para o nível de ansiedade-traço nível I (baixo) e nível II para ansiedadeestado.

Resultados similares já foram demonstrados, pessoas que possuem tipo cronobiológico indiferente, adaptam-se a qualquer horário e as do tipo vespertino suportam melhor ficarem acordadas de madrugada ${ }^{(6)}$.

As correlações nesta pesquisa acerca do tipo cronobiológico e turnos de trabalho obteve resultados que sustentam que existem algumas condições que são favoráveis para aceitação das pessoas aos turnos de trabalho, já estudados por alguns autores ${ }^{(6)}$, embora ainda sejam objetos de investigação. Nesta pesquisa, a maioria dos indivíduos está alocada nos turnos correspondentes aos cronótipos. Para o grupo do turno noturno a freqüência encontrada para o nível III de ansiedade-estado foi nula. A impressão que se tem sobre o turno noturno é de que suas características peculiares, quanto à atividade em horário não usual, são desgastantes, entretanto esta característica não pôde ser confirmada para o estado emocional.

A análise dos significados do trabalho noturno de onze enfermeiros, de cinco instituições de saúde, verificou que, além da necessidade econômica, o trabalho noturno apresentava pontos positivos, definidos pelos enfermeiros como: ter liderança, inexistência de competição acirrada, ficar longe de fofocas e, como conseqüência, menor desgaste e, também, maior autonomia ${ }^{(25)}$. Esses relatos foram ao encontro de nossas percepções, a respeito da permanência dos enfermeiros, em média, por dez anos, neste turno.

Referente aos níveis de ansiedade-traço, os escores não apresentaram diferenças significativas entre os turnos, para o grupo do turno matutino encontramos percentagem de maior valor para o nível II de ansiedade.

O cotidiano da profissão de enfermagem leva-nos a pensar que estes profissionais são extremamente ansiosos, por lidarem com aspectos emocionais do paciente-família, diante do sofrimento, dor e morte ${ }^{(9)}$. Somam-se a isto marcas de desgaste físico e mental, que aparecem sob forma de cansaço e sofrimento mental ${ }^{(4,26)}$. No entanto, verificou-se que houve um número reduzido de pessoas com escores para o nível III de ansiedade-estado (apenas dois sujeitos), sendo um do plantão matutino e o outro do plantão vespertino, para o mesmo nível de ansiedade-traço.

Esses achados apontam para uma seleção natural e contínua de indivíduos para os turnos aos quais mais se adaptam, conforme encontrado em estudo anterior ${ }^{(27)}$.

\section{CONCLUSÕES}

Os horários de trabalho, decorrentes das preferências individuais, estão em acordo com os cronótipo dos enfermeiros, o cronótipo mais freqüente foi o tipo matutino moderado.

Os indivíduos do turno matutino acordam mais cedo que os dos demais turnos e com ajuda do despertador. Em relação à duração do sono noturno, os enfermeiros do turno matutino dormem significativamente menos, em comparação com as pessoas do grupo vespertino e noturno.

Quanto à análise dos níveis de ansiedade-estado, concluiu-se que a maioria dos indivíduos, dos turnos matutino, vespertino e noturno, apresentou uma classificação de nível II (ansiedade moderada). Não obtivemos escores de ansiedade-estado de nível III (alto) para os enfermeiros no turno noturno.

Em relação aos níveis de ansiedade-traço, os turnos matutino e vespertino obtiveram escores para o nível II (moderado). Os enfermeiros do turno noturno foram classificados com escores nível I (baixo).

Ao estudarmos somente os enfermeiros, selecionando-os através de critérios de inclusões e exclusões pré-estabelecidos; objetivando homogeneizar a população, descobrimos após os estudos a importância destas condições de trabalho do enfermeiro, as quais acreditamos serem fundamentais para o bom exercício do trabalho.
Aspectos cronobiológicos do ciclo vigília-sono e níveis de ansiedade dos enfermeiros nos diferentes turnos de trabalho 
Maria Luiza Pesse Campos Milva Maria F. De Martino
Os profissionais do estudo, são enfermeiros assistenciais que realizam seu trabalho em sistema de turnos, possuindo somente um vínculo empregatício e, portanto, acreditamos com mais tempo para a atenção á família, ao lazer e ao cuidado pessoal.

Um diferencial deste estudo, realizado no Hospital de Clínicas da UNICAMP, é o sistema de turnos adotado por esta instituição, que talvez tenha contribuído para que os profissionais fossem menos ansiosos.

Os plantões com turnos fixos, não alternantes; diurno, com seis horas diárias de trabalho e dez folgas mensais, preservando folgas habituais mensais em dois finais de semana e, plantões noturnos, no mesmo sistema de turnos fixos, com 12 horas de trabalho e 60 horas de folga, são condições de trabalho privilegiadas perante outras instituições.

Os resultados apresentados permitirão que os dirigentes das instituições, da área de saúde, observem a importância de terem profissionais mais descansados, motivados e satisfeitos com suas condições de trabalho; refletindo numa melhor qualidade de cuidados a seus clientes e, levando-nos a projetar estas considerações, a todo âmbito hospitalar, extrapolando-os a outros trabalhadores em regime de turnos de trabalho. Acredita-se poder, com isto, contribuir para a melhoria do trabalho dos enfermeiros, a partir do entendimento e conhecimento das questões que interferem em seu cotidiano, propiciando campo para novas investigações.

\section{REFERÊNCIAS}

(1) Menna-Barreto L, Fortunato G. O que é cronobiologia? In: Cipolla-Neto J, Marques $\mathrm{N}$, Menna-Barreto LS, editores. Introdução ao estudo da cronobiologia. São Paulo: Ícone; 1988. p. 15-9.

(2) Afeche SC. Conceitos fundamentais da ritmicidade biológica. In: Cipolla Neto J, Marques N, Menna-Barreto LS, editores. Introdução ao estudo da cronobiologia. São Paulo: Ícone; 1988. p. 34-50.

(3) Menna-Barreto L. Relógios e rítmos. Cérebro Mente: Rev Eletron Div Ci Neurociência. [periódico online] 1997/1998; 4. Disponível em: http://www.cerebromente.org.br/n04/ mente/cloks.htm (15 out. 2001)

(4) Ferreira LL. Trabalho em turnos: temas para discussão. Rev Bras Saúde Ocup 1987; 15(58):27-32.

(5) Ferreira LL. Aplicações da cronobiologia na organização do trabalho humano. In: CipollaNeto J, Marques N, Menna-Barreto LS, editores. Introdução ao estudo da cronobiologia. São Paulo: Ícone; 1988. p. 233-52.

(6) Rutenfranz J, Knauth P, Fisher FM. Trabalho em turnos e noturno. São Paulo: Hucitec; 1989.

(7) Costa G. The problem: shiftwork. Chronob Intern 1997; 2:89-98.

(8) Fisher FM, Lieber RR, Brown FM. Trabalho em turnos e as relações com a saúde-doença. In: Mendes R, editor. Patologia do trabalho. Rio de Janeiro: Atheneu; 1995.
(9) Menzies I. O funcionamento das organizações como sistemas sociais de defesa contra a ansiedade. São Paulo: Escola de Administração de Empresas/FGV; 1970./mimeo/.

(10) Spielberger CD, Gorsuch RL Lushene RE. Inventário de Ansiedade Traço-Estado IDATE. Rio de Janeiro: CEPA; 1979.

(11) May R. O homem a procura de sí mesmo. 23 ed. Petrópolis: Vozes; 1996.

(12) Harma H. Individual differences in tolerance to shift work: a review. Ergonomics 1993; 1(3):101-9.

(13) Barton J. Choosing to work at night: a moderating influence on individual tolerance to shift work. J Appl Psychol 1994; 79(3):449-54.

(14) Delafosse JY, Leger D, Quera-Salva MA, Samson O, Adrien J. Comparative study of actigraphy and ambulatory polysomnography in the assessment of adaptation to night shift work in nurses. Rev Neurol 2000; 56(6/7):641-5.

(15) Verhaegen P, Cober R, De Smedt M, Dirkx J, Kerstens J, Ryvers D et al. The adaptation of night nurses to different work schedules. Ergonomics 1987; 30(9):1301-9.

(16) De Martino MMF. Estudo da variabilidade cicardiana da temperatura oral, do cíclio vigília-sono e de testes psicológicos de enfermeiras em diferentes turnos de trabalho. [tese]. Campinas (SP): Universidade Estadual de Campinas; 1996. 
(17) Horne JA, Ostberg O. A self-assessment questionnaire to determine morningnesseveningness in human circadian rhytms. Int $\mathbf{J}$ Chronobiol 1976; 4(2):97-110.

(18) Campos MLP, De Martino MMF. Estudo das características cronobiológicas dos enfermeiros docentes: cronótipo. Nursing 2001; 4(42):31-4.(edição brasileira)

(19) Benedito-Silva AA, Menna-Barreto L, Marques N, Tenreiro S. A self-assessment questionnaire for the determination of morningness-eveningness types in Brazil. In: Hayes DK, Pauly JE, Reiter RJ, editors. Chronobiology: its role in clinical medicine, general biology and agriculture, part B. New York: Wiley-Liss; 1990. p. 89-98.

(20) Biaggio AMB, Natalício L, Spielberger CD. Desenvolvimento da forma experimental em português do Inventário de Ansiedade Traço-Estado (IDATE) de Spielberger. Arq Bras Psicol Apl 1977; 3:31-44.

(21) Andrade M. Ciclio vigília-sono de adolescentes: um estudo longitudinal. [dissertação] São Paulo (SP): Universidade de São Paulo; 1991.
(22) Bulhões I. Riscos do trabalho em enfermagem. $2^{\mathrm{a}}$ ed. Rio de Janeiro: Folha Carioca; 1998.

(23) Stampi C. Cochilos e padrões polifásicos do sono humano. In: Reimão R. Sono: estudo abrangente. São Paulo: Atheneu; 1996. p. $392-411$.

(24) Dinges DF. Adult napping and its effects on ability to function. In: Stampi C, editor. Why nap? Boston: Birkhauser; 1992. p. 118-34.

(25) Pontes Z. O trabalho noturno do enfermeiro: busca de significados sobre o repouso antes, durante e após o plantão. Rev Bras Enferm 1992; 45(1):80-7.

(26) Pitta A. Hospital: dor e morte como ofício. $3^{\mathrm{a}}$ ed. São Paulo: Hucitec; 1994

(27) Chaves EC. Stress e trabalho do enfermeiro: a influência de características individuais no ajustamento e tolerância ao turno noturno. [tese]. São Paulo (SP): Instituto de Psicologia da USP; 1994.
Aspectos cronobiológicos do ciclo vigília-sono e níveis de ansiedade dos enfermeiros nos diferentes turnos de trabalho 